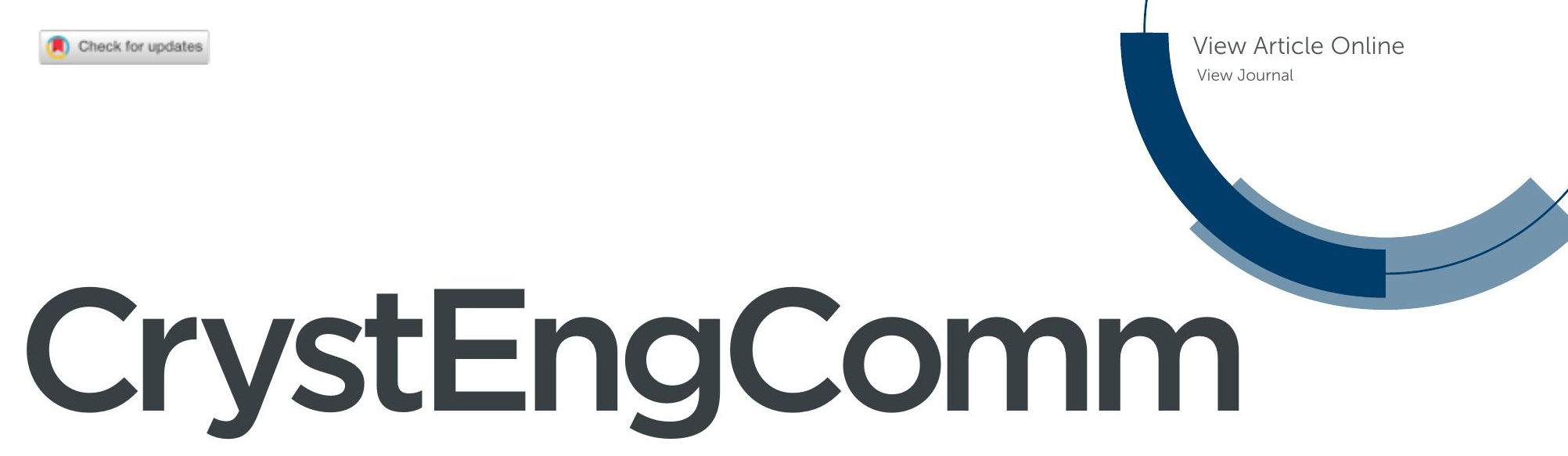

Accepted Manuscript

This article can be cited before page numbers have been issued, to do this please use: F. Pereira,

CrystEngComm, 2020, DOI: 10.1039/D0CE00070A.
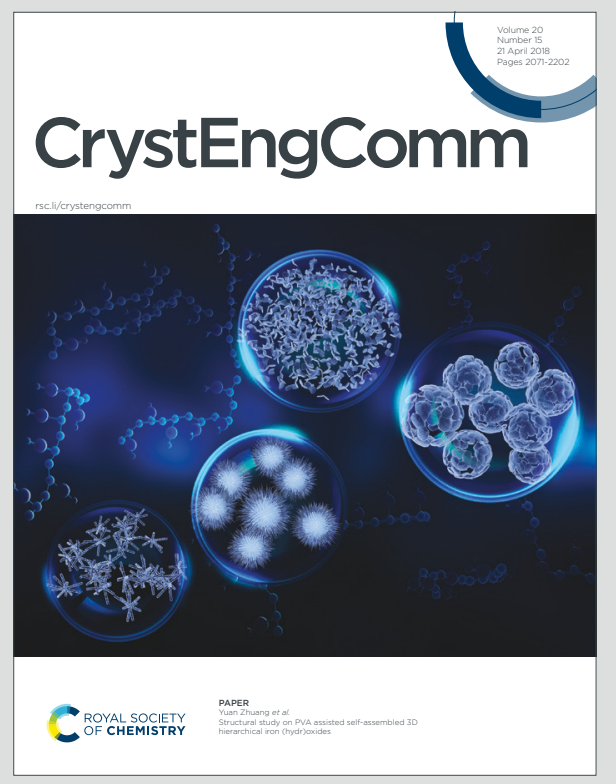

This is an Accepted Manuscript, which has been through the Royal Society of Chemistry peer review process and has been accepted for publication.

Accepted Manuscripts are published online shortly after acceptance, before technical editing, formatting and proof reading. Using this free service, authors can make their results available to the community, in citable form, before we publish the edited article. We will replace this Accepted Manuscript with the edited and formatted Advance Article as soon as it is available.

You can find more information about Accepted Manuscripts in the Information for Authors.

Please note that technical editing may introduce minor changes to the text and/or graphics, which may alter content. The journal's standard Terms \& Conditions and the Ethical guidelines still apply. In no event shall the Royal Society of Chemistry be held responsible for any errors or omissions in this Accepted Manuscript or any consequences arising from the use of any information it contains. 


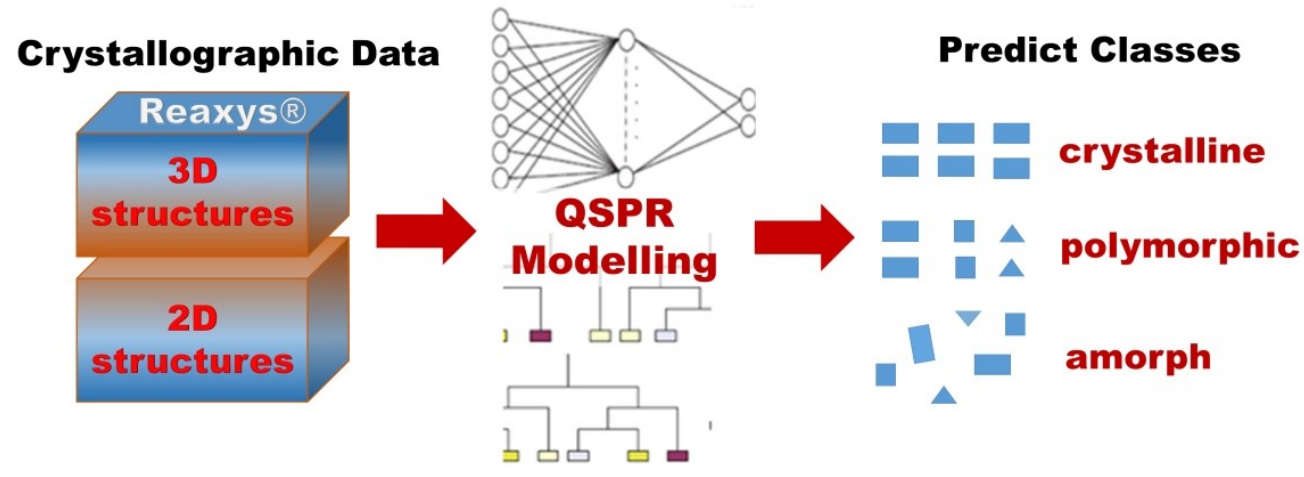




\section{Machine learning methods to predict the crystallization propensity of small organic molecules}

Received 00th January 20xx, Accepted 00th January 20xx

DOI: $10.1039 / x 0 \times x 00000 x$

\begin{abstract}
Florbela Pereira*
Machine learning $(\mathrm{ML})$ algorithms were explored for the prediction of the crystallization propensity based on molecular descriptors and fingerprints generated from 2D chemical structures and 3D molecular descriptors from 3D chemical structures optimized with empirical methods. In total 57,815 molecules were retrieved from the Reaxys ${ }^{\circledR}$ database, from those 53,998 molecules are recorded as crystallines (A class), 3,097 polymorphics (B class), and 720 as amorphs (C class). A training data set with 40,462 organic molecules was used to build the models, which were validated with an external test set comprising 17,353 organic molecules. Several ML algorithms such as Random Forest (RF), Support Vector Machines (SVM), and deep learning Multilayer Perceptron networks (MLP) were screened. The best performance was achieved with a consensus classification model obtained by RF, SVM, and MLP models, which predicted the external test set with an overall predictive accuracy $(\mathrm{Q})$ up to $80 \%$.
\end{abstract}

\section{Introduction}

The predicting the crystallization propensity of small organic molecules is one of the most significant challenges facing the chemical, pharmaceutical, energy and food industry today. ${ }^{1-3}$ Although the experimental conditions such as temperature, solvent polarity, molar concentration that were used might influence the crystallization process, there have been a high degree of agreement between the crystallizability ranking using various methods of sample preparation, and the authors in several works concluded that the crystallization tendency is dominated by the physicochemical properties of the active pharmaceutical ingredient, with the solidification process being less important. ${ }^{2}$, 4-7 Therefore the prediction of the crystallization propensity taking in account the chemical structure of small organic molecules can be done and can be very useful mainly in two circumstances when predictions of this type are needed. First, predicting non-crystalline molecules, it is very relevant in absorption, distribution, metabolism, excretion (ADME) properties of pharmaceutical compounds, ${ }^{1,2}$ as well as organic photo-voltaic cells. ${ }^{3}$ In general, an amorphous drug substance or formulation can increase the instantaneous solubility and promote greater oral absorption as compared with crystalline ones and hence crystallization can be an important mode of failure and can lead to compromised in vivo drug performance. ${ }^{2}$ Second, predicting crystalline molecules, it is very important when a drug substance is first synthesized, and

LAQV and REQUIMTE, Departamento de Química, Faculdade de Ciências e Tecnologia, Universidade Nova de Lisboa, Caparica, Portugal. E-mail: florbela.pereira@fct.unl.pt

+ Footnotes relating to the title and/or authors should appear here.

Electronic Supplementary Information (ESI) available: [details of any supplementary information available should be included here]. See DOI: 10.1039/x0xx00000x a stable crystal for use in early clinical or toxicology formulations is need. Understanding which chemical structural features (polarity, molecular size, flexibility, etc.) of small organic molecules are more relevant for their crystallisability is important for several fields, however, there has been very little published work focused on this topic. Typically, crystalline molecules tend to be of low molecular weight (MW), ${ }^{2,8}$ simple structures with fewer number of rotatable bonds (NRotB) compared to non-crystalline molecules, $1,2,8$ which are usually more structurally complex. Flexible molecules (i.e., higher number of rotatable bonds) introduce potential complications to the crystallization process since they can exist in solution (or melt) as energetically similar conformers, and the crystallization process must choose between conformers, giving rise to polymorphs a complex procedure not encountered by rigid molecules. ${ }^{8,9}$

In the last years there are a several reports of machine learning $(\mathrm{ML})$ modelling to predict the crystallization propensity of proteins, ${ }^{10}$ however only few works were reported for small organic molecules. ${ }^{1,11,12}$ Wicker \& Cooper $^{1}$ developed the most extended published work for $\mathrm{ML}$ modelling to predict the crystallization propensity of small organic molecules with almost 36,000 molecules, 17,920 non-crystalline and 17,938 crystalline molecules. The best model achieved an overall predictive accuracy (Q) up to $90 \%$, however in this study the molecules belonging to the non-crystalline class were only set in this way because they did not appear in the Cambridge Structural Database (CSD), contrary to what was done in the current work. Bhardwaj et al. ${ }^{11}$ used a library of substituted acylanilides comprising 382 molecules, which their experimental crystallization propensities were determined and published, for building the quantitative structure-property relationship (QSPR) model. The random forest (RF) model built were able to predict the crystallisability of acylanilide 
derivatives with approximately $70 \%$ accuracy. Pillong et al. ${ }^{12}$ reported a different, complementary approach, using the crystallization outcomes for 319 publicly available compounds in up to 18 different solvents spread over 5710 individual single solvent evaporation trials. The authors argued that the ML models developed using the RF algorithm can be used to guide the solvent choice when crystallizing a given organic compound.

Here we report the exploration of $M L$ tools for predicting crystallization propensity of small organic molecules, using a data set retrieved from the Reaxys ${ }^{\circledR}$ (https://www.reaxys.com) database and comprising 57,815 molecules, from those 53,998 molecules are recorded as crystallines (A class), 3,097 polymorphics (B class), and 720 as amorphs (C class). Three data mining methods Random Forest (RF), Support Vector Machine (SVM), deep learning Multilayer Perceptron networks (dMLP) were explored to build classification models for crystallization propensity prediction. The performances of the models were successfully evaluated by internal validation and external test set validation, achieving a $\mathrm{Q}$ of $84 \%$ and $80 \%$ for training and test sets, respectively.

\section{Methods}

\subsection{Data Sets/Selection of Training and Test Sets}

Molecular structures were retrieved from the Reaxys ${ }^{\circledR}$ database (Elsevier Information Systems $\mathrm{GmbH}$ ) and were filtered with following items: $98 \mathrm{~g} / \mathrm{mol}<\mathrm{MW}<1080 \mathrm{~g} / \mathrm{mol}$; only one or two fragments; PubChem database; exclude molecules with the $\mathrm{B}$, $\mathrm{P}, \mathrm{Si}, \mathrm{Te}, \mathrm{Ag}, \mathrm{Hg}, \mathrm{Bi}, \mathrm{Na}$, and $\mathrm{K}$ atoms, searching by crystal data and their chemical structures saved in SMILES data format. In total 57,815 molecules were retrieved, from those 53,998 molecules are recorded as crystallines (A class), 3,097 polymorphics (B class), and 720 as amorphs (C class). It is important to note that there are 812 molecules belonging only to class B that are also duplicates of class A (i.e. the 812 duplicate molecules were removed from class $A$ and maintained in class $B$ ). The data set was randomly divided into a training set of 40,462 molecules (A class: 37,790 molecules, B class: 2,167 molecules, and C class: 505 molecules), and a test set of 17,353 molecules (A class: 16,208 molecules, B class: 930 molecules, and C class: 215 molecules), a partition of approximately 70:30 for the training and test sets. Two other external data sets (test set I and test set II) were also compiled from the work reported by Bhardwaj et al. ${ }^{11}$ and from the PubChem database, which comprising a library of 402 substituted acylanilide derivatives with experimental crystallization propensities ${ }^{13}$ and a library of 125 substances from Crystallography Open Database (http://www.crystallography.net/), respectively. The test set I contains 205 acylanilide derivatives of A class and 197 acylanilide derivatives of B or C class. For class A were considered only the acylanilide derivatives reported with crystal structure obtained. ${ }^{13}$ For class B or C were considered all acylanilide derivatives reported as synthesized, but not yet crystallized and with wrong crystal structure obtained, as well as those awaiting crystal structure determination. ${ }^{13}$

\subsection{Calculation of Molecular Descriptors and Fingerprints $s_{\text {rticle }}$ Online} The molecular structures were standardized by normalizing tautomeric and mesomeric groups and by removing small disconnected fragments using JChem Standardizer tool version 5.7.13.0 (ChemAxon Ltd., Budapest, Hungary). Threedimensional models of the molecular structures were generated with CORINA version 2.4 (Molecular Networks $\mathrm{GmbH}$, Erlangen, Germany). Empirical molecular fingerprints and 1D\&2D molecular descriptors were calculated by PaDELDescriptor version 2.21 (Yap Chun Wei, Pharmaceutical Data Exploration Laboratory). ${ }^{14}$ Different types of fingerprints with different sizes were calculated and explored: 166 MACCS (MACCS keys), 307 Substructure (presence and count of SMARTS patterns for Laggner functional group classificationSub and SubC respectively), 881 PubChem fingerprints (ftp://ftp.ncbi.nlm.nih.gov/pubchem/specifications/pubchem_ fingerprints.txt), 1024 CDK (circular fingerprints), and 1024 CDK extended (Ext circular fingerprints with additional bits describing ring features). The 1D\&2D molecular descriptors comprise 1443 descriptors, including electronic, topological, and constitutional descriptors. Radial Distribution Function (RDF) pair descriptors, ${ }^{15}$ 3D RDF descriptors, were calculated by sampling the function of Equation (1) at 30 equally distributed values of $r$ between 0 and $3.0 \AA$ :

$$
R D F(r)=\sum_{i=1}^{N-1} \sum_{j=1+1}^{N} p_{i} p_{j} e^{-B\left(r-r_{i j}\right)^{2}}
$$

where $\mathrm{N}$ is the number of atoms in the molecule, $\mathrm{p}_{i}$ is the charge of atom $i, \mathrm{~B}$ is a fuzziness parameter (it was 100 in this study), and $r_{i j}$ is the 3D distance between atoms $i$ and $j$. Three sets of 30 RDF descriptors were separately calculated, derived from atom pairs with (a) a positive and a negative charge, (b) two positive charges, and (c) two negative charges. The partial atomic charges-natural bond orbital (NBO) partial atomic charges were estimated using a ML tool developed by Aires-de-Sousa and co-workers (http://joao.airesdesousa.com/charges). ${ }^{16}$

\subsection{Selection of descriptors}

In the quest for QSPR models with the minimum possible number of descriptors, descriptor selection was performed based on the importance of descriptors assessed by RF (mean decrease in accuracy measure) with $\mathrm{R}$ program version 3.6.1 ${ }^{17}$ and CFS (Correlation-based Feature Subset Selection) ${ }^{18}$ algorithm implemented in Weka 3.8.3.19 In CFS, the heuristic takes into account the usefulness of individual descriptors for predicting the crystallization propensity together with the level of intercorrelation among them. The experiments were conducted with the AttributeSelectedClassifier routine of Weka with CfsSubsetEval option for attribute evaluator and BestFirst or PSOSearch option as the search method.

\subsection{Class balancer}

In general, the class imbalance introduces a bias in the performance of the ML algorithms due to their preference towards the majority class. ${ }^{20}$ Our crystallization propensity dataset is very unbalanced, and the imbalance ratio is 19:1 and $80: 1$ for the crystalline/polymorphic and crystalline/amorphous 
pair classes, respectively. In order to address this issue, three approaches using a base-classifier with RF in R program version 3.6.1, ${ }^{17}$ the CostSensitiveClassifier in Weka version 3.8.3, ${ }^{19}$ and the class_weight parameters in Keras version 2.2.5 with some $M L$ techniques were intended. The sampsize is the parameter in the RF with $\mathrm{R}$ specified for balancing the classes. This parameter was set to be of the same size as the minority class (C class). With this parameter, some molecules belonging to the minority class were used more than once. The CostSensitiveClassifier was used to introduce cost-sensitivity reweighting training molecules according to the total cost assigned to each class, using the following weights $\{A: 1 ; B: 17$; C: 75$\}.{ }^{20}$ The class_weight is optional dictionary mapping class indices to a weight value, used for weighting the loss function (during training only) and therefore can be seen as useful way to tell the model to "pay more attention" to molecules from an under-represented classes, using the following weights $\{A: 1 ; B$ : 34; C: 75\}.

\subsection{ML methods}

\subsubsection{Reduces Error Pruning Tree (REPTree)}

The decision tree was built using information gain and prunes it using reduced-error pruning (with back fitting). A fast decision tree learner was built with Weka version 3.8.3, using the REPTree algorithm for the training set in ten-fold crossvalidation using 10 molecules in a leaf (minNum parameter), the maximum tree depth of 10 (maxDepth parameter) and with pruning.

\subsubsection{Random forests (RF)}

A $\mathrm{RF}^{21,22}$ is implemented as an ensemble of unpruned classification trees which are created using bootstrap samples of the training set. For each individual tree, the best split at each node is defined using a randomly selected subset of descriptors. Each individual tree is created using a different training and validation set. Prediction is made by a majority vote of the classification trees in the forest. Performance is internally assessed with the prediction error for the objects left out in the bootstrap procedure (internal cross-validation or OOB estimation). The method quantifies the importance of a descriptor by the increase in misclassification occurring when the values of the descriptor are randomly permuted, correlated with the mean decrease in accuracy parameter. RFs also assign a probability to every prediction based on the number of votes obtained by the predicted class. RFs were grown with the $\mathrm{R}$ program, ${ }^{17}$ version 3.6.1, using the Random Forest library. ${ }^{23}$ As a result of the nature of three-class imbalance, this problem was alleviated setting the class weights ranges between 1-500; 1 500; and 1-500 for A, B, and C classes, respectively, using the sampsize parameter.

\subsubsection{Support Vector Machine (SVM)}

$\mathrm{SVM}^{24}$ map the data into a hyperspace through a nonlinear mapping (a boundary or hyperplane) and then separate the three classes of molecules in this space. The boundary is positioned using examples in the training set that are known as the support vectors. With nonlinear data, kernel functions can be used to transform it into a hyperspace where the classes become linearly separable. In this study, SVM were established with Weka version 3.8.3, using the LIBSVAPpalekage.(250 Theotype of SVM was set to C-SVM-classification and the kernel function was the radial basis function. The parameter $\mathrm{C}$ of the C-SVMclassification was optimized in the range of $10-500$, and the default $\gamma$ parameter in the kernel function was used. The weights of the three classes were set to $\{A: 1 ; B: 17 ; C: 75\}$.

\subsubsection{Deep learning Multilayer Perceptron networks (dMLP)}

The open-source software library Keras ${ }^{26}$ version 2.2 .5 based on Tensorflow numerical backend engine ${ }^{27}$ was used to implement feed-forward neural networks. These popular software tools, written in Python, make development and application of deep neural networks relatively easy, however the major challenge in the application of ${ }_{d} \mathrm{MLP}$ is the design of a proper network architecture. After several experiments the final optimal hyperparameter settings were selected for our study based in 10-fold cross-validation experiments with the training set and were listed in Table 1.

Table 1 Hyper-parameter settings of the best ${ }_{d}$ MLP model

\begin{tabular}{lc}
\hline Hyper-parameter & Setting \\
\hline Initializer & Glorot \\
Number of hidden layers & uniform \\
Number of neurons in the 1st and 2nd layer & 2 \\
Number of neurons in the 3rd layer & 321 \\
Activation 1st and 2nd layer & 3 \\
Activation 3rd layer & Relu \\
Batch size & Softmax \\
Optimizer & 36 \\
Loss & Adam \\
Epochs & Categorical \\
& crossentropy \\
\hline
\end{tabular}

\section{Results and discussion}

Two wide sets of descriptors were explored, one with 6 different types of fingerprints (FPs) with different sizes (166 MACCS, MACCS keys; 307 Substructure, presence and count Sub and SubC respectively; 881 PubChem FPs; 1024 CDK, circular FPs; and 1024 CDK Ext, extended circular FPs with additional bits describing ring features) and other with a total of 1443 1D\&2D molecular descriptors (including electronic, topological, and constitutional descriptors). The FPs and the molecular descriptors were calculated by PaDEL-Descriptor version 2.21 (Yap Chun Wei, Pharmaceutical Data Exploration Laboratory). ${ }^{14}$ RF ML technique was used for building the crystallization propensity classification models, and the performance of the models was successfully evaluated by internal validation (out-of-bag, OOB, estimation on the training set), Table 2. The best models reported in Table 2 (SubC, PubChem and 1D\&2D) considering the G-Mean value were used to evaluate the performance of 3D descriptors such as RDF descriptors using a range of 30 and partial atomic charge as atomic property. Since the classes are unbalanced, criterions other than G-Mean were also stipulated, i.e. SP_B $\geq 0.4$ and $S P \_C \geq 0.4$. The four set of descriptors (RDF, 


\section{ARTICLE}

SubC + RDF, PubChem + RDF and 1D\&2D + RDF) were explored for modelling the crystallisability of organic molecules using the RF algorithm, in Table 3 were presented the results for the training and test sets.

Table 2 Evaluation of the predictive performance of FPs and 1D\&2D molecular descriptors for modelling the crystallisability of organic molecules using the RF algorithm for the training set in an OOB estimation

\begin{tabular}{|c|c|c|c|c|c|c|c|}
\hline Model & MACCS & Sub & Subc & PubChem & CDK & ExtCDK & 1D\&2D \\
\hline $\mathrm{TA}^{\mathrm{a}}$ & 30266 & 29845 & 32131 & 31439 & 31565 & 32058 & 32627 \\
\hline $\mathrm{TB}^{\mathrm{b}}$ & 1016 & 1149 & 1038 & 1117 & 1050 & 1063 & 947 \\
\hline $\mathrm{TC}^{\mathrm{c}}$ & 120 & 160 & 208 & 215 & 171 & 162 & 245 \\
\hline $\mathrm{FAB}^{\mathrm{d}}$ & 889 & 963 & 1057 & 982 & 1080 & 1069 & 1097 \\
\hline $\mathrm{FAC}^{\mathrm{e}}$ & 209 & 194 & 220 & 202 & 262 & 277 & 218 \\
\hline $\mathrm{FBA}^{f}$ & 5186 & 6804 & 4441 & 5062 & 5346 & 4887 & 3444 \\
\hline $\mathrm{FBC}^{\mathrm{g}}$ & 176 & 151 & 77 & 88 & 72 & 66 & 42 \\
\hline $\mathrm{FCA}^{\mathrm{h}}$ & 2338 & 1141 & 1218 & 1289 & 879 & 845 & 1719 \\
\hline $\mathrm{FCB}^{\mathrm{i}}$ & 262 & 55 & 72 & 68 & 37 & 35 & 123 \\
\hline $\mathrm{SE}^{\mathrm{j}}$ & 0.801 & 0.790 & 0.850 & 0.832 & 0.835 & 0.848 & 0.863 \\
\hline SP_B' & 0.469 & 0.530 & 0.479 & 0.516 & 0.485 & 0.491 & 0.437 \\
\hline SP_Cm & 0.238 & 0.317 & 0.412 & 0.426 & 0.339 & 0.321 & 0.485 \\
\hline$Q^{n}$ & 0.776 & 0.770 & 0.825 & 0.810 & 0.810 & 0.823 & 0.836 \\
\hline G-Meanº & 0.418 & 0.447 & 0.481 & 0.480 & 0.469 & 0.473 & 0.485 \\
\hline
\end{tabular}

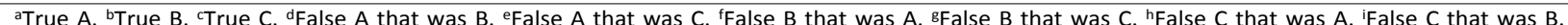
jSensitivity, the ratio of true $A$ to the sum of true $A$ and false $A$. 'Specificity $B$, the ratio of true $B$ to the sum of true $B$ and false $B$. mSpecificity $C$, the ratio of true $C$ to the sum of true $C$ and false $C$. nOverall predictive accuracy, the ratio of the sum of true $A$, true $B$ and true $C$ to the sum of true $A$, true $B$, true $\mathrm{C}$, false $\mathrm{A}$, false $\mathrm{B}$ and false $\mathrm{C}$. ${ }^{\circ} \mathrm{G}$-mean, the average of $\mathrm{G}$-mean $\mathrm{A}, \mathrm{G}$-mean $\mathrm{B}$ and $\mathrm{G}$-mean $\mathrm{C}$, where $\mathrm{G}$-mean $\mathrm{x}$ is the square root of the product of precision $x$ and recall $x$.

Table 3 Evaluation of the predictive performance of RDF descriptors for modelling the crystallisability of organic molecules using the RF algorithm

\begin{tabular}{lccccc}
\hline Model & SE $^{\mathbf{a}}$ & SP_B $^{\mathbf{b}}$ & SP_C$^{\mathbf{c}}$ & Q $^{\mathbf{d}}$ & $\begin{array}{l}\text { G- } \\
\text { Mean }^{\mathbf{e}}\end{array}$ \\
\hline \multicolumn{7}{c}{ Training Set (OOB) } \\
\hline RDF & 0.791 & 0.464 & 0.517 & 0.770 & 0.451 \\
SubC_RDF & 0.841 & 0.434 & 0.503 & 0.815 & 0.471 \\
PubChem_RDF & 0.847 & 0.442 & 0.515 & 0.821 & 0.479 \\
1D_2D_RDF & 0.864 & 0.430 & 0.505 & 0.836 & $\mathbf{0 . 4 8 6}$ \\
\hline \multicolumn{7}{c}{ Test Set } \\
\hline RDF & 0.791 & 0.468 & 0.484 & 0.770 & 0.448 \\
SubC_RDF & 0.842 & 0.431 & 0.465 & 0.815 & 0.466 \\
PubChem_RDF & 0.849 & 0.451 & 0.479 & 0.823 & 0.477 \\
1D_2D_RDF & 0.866 & 0.428 & 0.428 & 0.837 & 0.475 \\
\hline
\end{tabular}

aSensitivity, the ratio of true $A$ to the sum of true $A$ and false $A$. ${ }^{b}$ Specificity $B$, the ratio of true $B$ to the sum of true $B$ and false $B$. 'Specificity $C$, the ratio of true $C$ to the sum of true $C$ and false $C$. ${ }^{d}$ Overall predictive accuracy, the ratio of the sum of true $A$, true $B$ and true $C$ to the sum of true $A$, true $B$, true $C$, false $A$, false $B$ and false $C$. ${ }^{~} G$-mean, the average of G-mean A, G-mean B and G-mean C, where $\mathrm{G}$-mean $x$ is the square root of the product of precision $x$ and recall $x$.

For the best models for the training set, 1D\&2D + RDF descriptors (Table 2), was evaluated the descriptor selection based on the importance assigned by RF model with R program and by the CFS algorithm implemented in Weka - Table 4. The 321 descriptors selected by the CFS algorithm of 1D\&2D + RDF model were identified by the RF algorithm and enabled the training of a new RF model with better prediction accuracy in accordance with G-Mean value than the model trained with the whole set of descriptors (Table 2).

A comparison of three $\mathrm{ML}$ techniques using the Weka software (SVM), R software (RF), and Keras software ( $\left.{ }_{d} M L P\right)$ for building the crystallisability of organic molecules models with the 321 descriptors that were selected in the CfsSubsetEval routine is shown in Table 5. The selected 321 descriptors included 298 1D\&2D (183 topological and 120 count type descriptors) and 23 RDF 3D descriptors ( 7 of type $a, 7$ of type $b$, and 9 of type $c$ ). The 1D\&2D descriptors comprise 68 autocorrelation topological descriptors, which are 45 Broto-Moreau, 14 Moran, and 9 Geary autocorrelation descriptors, weighted by mass, charges, van der Waals volumes, Sanderson electronegativities, polarizabilities, first ionization potential or I-state. Other topological descriptors such as 34 Barysz matrix, 13 Burden modified eigenvalues, 6 Detour matrix, 15 extended topochemical atom, 8 topological charge, and 4 walk count descriptors were also presented. 
Table 4 Exploration of descriptor selection using RF algorithm with 1D\&2D + RDF descriptors

\begin{tabular}{|c|c|c|c|c|c|}
\hline Descriptors & $\mathrm{SE}^{\mathrm{a}}$ & SP_B ${ }^{b}$ & SP_Cc & $\mathbf{Q}^{\mathbf{d}}$ & $\begin{array}{l}\text { G- } \\
\text { Meane }\end{array}$ \\
\hline \multicolumn{6}{|c|}{ Training Set (OOB) } \\
\hline $50^{f}$ & 0.846 & 0.438 & 0.517 & 0.820 & 0.476 \\
\hline $100^{f}$ & 0.840 & 0.450 & 0.515 & 0.815 & 0.476 \\
\hline $150^{f}$ & 0.845 & 0.445 & 0.515 & 0.819 & 0.478 \\
\hline $200^{f}$ & 0.842 & 0.459 & 0.517 & 0.818 & 0.480 \\
\hline $250^{f}$ & 0.844 & 0.448 & 0.527 & 0.819 & 0.479 \\
\hline 399 & 0.793 & 0.565 & 0.602 & 0.778 & 0.483 \\
\hline $321^{\mathrm{h}}$ & 0.812 & 0.561 & 0.604 & 0.796 & 0.493 \\
\hline \multicolumn{6}{|c|}{ Test Set } \\
\hline $50^{f}$ & 0.841 & 0.441 & 0.461 & 0.815 & 0.466 \\
\hline $100^{f}$ & 0.842 & 0.453 & 0.451 & 0.816 & 0.468 \\
\hline $150^{f}$ & 0.848 & 0.447 & 0.442 & 0.821 & 0.469 \\
\hline $200^{f}$ & 0.844 & 0.440 & 0.437 & 0.817 & 0.465 \\
\hline $250^{f}$ & 0.843 & 0.443 & 0.470 & 0.817 & 0.469 \\
\hline 399 & 0.796 & 0.565 & 0.554 & 0.780 & 0.478 \\
\hline $321^{\mathrm{h}}$ & 0.816 & 0.558 & 0.554 & 0.798 & 0.488 \\
\hline
\end{tabular}

aSensitivity, the ratio of true $A$ to the sum of true $A$ and false $A$. ${ }^{b}$ Specificity $B$, the ratio of true $B$ to the sum of true $B$ and false $B$. 'Specificity $C$, the ratio of true $C$ to the sum of true $C$ and false $C$. 'Overall predictive accuracy, the ratio of the sum of true $A$, true $B$ and true $C$ to the sum of true $A$, true $B$, true $C$, false $A$, false $B$ and false $C$. ' $G$-mean, the average of G-mean A, G-mean B and Gmean $C$, where $G$-mean $x$ is the square root of the product of precision $x$ and recall $x .{ }^{f}$ The most important descriptors in accordance with mean decrease in accuracy. ${ }^{8 S}$ elected using CfsSubsetEval option for attribute evaluator and the Best First option as the search method. hSelected using CfsSubsetEval option for attribute evaluator and the PSOSearch option as the search method.

Table 5 Exploration of different ML algorithm using the 321 selected descriptors

\begin{tabular}{|c|c|c|c|c|c|}
\hline ML & $\mathbf{S E}^{\mathrm{a}}$ & SP_B ${ }^{b}$ & SP_Cc & $\mathbf{Q}^{\mathbf{d}}$ & $\begin{array}{l}\text { G- } \\
\text { Meane }\end{array}$ \\
\hline \multicolumn{6}{|c|}{ Training Set } \\
\hline RF & 0.812 & 0.561 & 0.604 & 0.796 & 0.493 \\
\hline SVM & 0.732 & 0.596 & 0.695 & 0.724 & 0.466 \\
\hline MLP & 0.807 & 0.507 & 0.788 & 0.790 & 0.503 \\
\hline \multicolumn{6}{|c|}{ Test Set } \\
\hline RF & 0.816 & 0.558 & 0.554 & 0.798 & 0.488 \\
\hline SVM & 0.727 & 0.593 & 0.674 & 0.719 & 0.460 \\
\hline MLP & 0.801 & 0.475 & 0.540 & 0.781 & 0.461 \\
\hline \multicolumn{6}{|c|}{$\begin{array}{l}\text { aSensitivity, the ratio of true } A \text { to the sum of true } A \text { and false } A \text {. }{ }^{b} \text { Specificity } \\
B \text {, the ratio of true } B \text { to the sum of true } B \text { and false } B \text {. cSpecificity } C \text {, the ratio } \\
\text { of true } C \text { to the sum of true } C \text { and false } C \text {. dOverall predictive accuracy, the } \\
\text { ratio of the sum of true } A \text {, true } B \text { and true } C \text { to the sum of true } A \text {, true } B \text {, } \\
\text { true } C \text {, false } A \text {, false } B \text { and false } C \text {. e } G \text {-mean, the average of } G \text {-mean } A, G- \\
\text { mean } B \text { and } G \text {-mean } C \text {, where } G \text {-mean } x \text { is the square root of the product of } \\
\text { precision } x \text { and recall } x \text {. }\end{array}$} \\
\hline
\end{tabular}

Taking into consideration the 20 most important descriptors from the 321 selected descriptors in the RF model, that was reported in Table 5, eighteen are 1D\&2D descriptors and two are RDF descriptors. The RDF descriptors of type a and $c$, RDF_np_4 and RDF_nn_16, are the first and fifth most important descriptors respectively, followed by two topological charge descriptor (JGI3 and JGI4, mean topological charge index of order 3 and $4,2^{\text {nd }}$ and $17^{\text {th }}$, respectively), a molecular linear free energy relation (MLFER) descriptor (MLFER_A, overall or summation solute hydrogen bond acidity, $3^{\text {rd }}$ ), and six autocorrelation topological descriptors - AATS2V Artideroto- $^{\text {(B) }}$ Moreau autocorrelation - lag 2 / weighĐed by 1 WrandDeECARZals volumes, $4^{\text {th }}$ ), MATS1i (Moran autocorrelation - lag 1 / weighted by first ionization potential, $6^{\text {th }}$ ), GATS2 $v$ (Geary autocorrelation - lag 2 / weighted by van der Waals volumes, $11^{\text {th }}$ ), GATS3m (Geary autocorrelation - lag 3 / weighted by mass, $12^{\text {th }}$ ), ATSC2v (centered Broto-Moreau autocorrelation - lag 2 / weighted by van der Waals volumes, $19^{\text {th }}$ ), and AATS1p (average BrotoMoreau autocorrelation - lag 1 / weighted by polarizabilities, $\left.20^{\text {th }}\right)$. Besides these descriptors, there are three count atom type electrotopological state descriptors, $\mathrm{nsOH}$ (count of atomtype E-State: $-\mathrm{OH}, 7^{\text {th }}$ ), SHssNH (Sum of atom-type H E-State: $\mathrm{NH}-, 9^{\text {th }}$ ) and maxHBint6 (maximum E-State descriptors of strength for potential hydrogen bonds of path length $6,18^{\text {th }}$ ), one constitutional descriptor, CrippenLogP (Crippen's LogP, $8^{\text {th }}$ ), one topological descriptor, topoDiameter (topological diameter, $\left.10^{\text {th }}\right)$, three information content descriptors, CIC1 (complementary information content index, neighborhood symmetry of 1 -order, $13^{\text {th }}$ ), BIC1 (bond information content index, neighborhood symmetry of 1-order, $\left.15^{\text {th }}\right)$, and MIC2 (modified information content index, neighborhood symmetry of 2-order, 16 $6^{\text {th }}$ ), and finally an extended topochemical atom descriptor, ETA_Epsilon_5 (a measure of electronegative atom count, $\left.14^{\text {th }}\right)$.

Furthermore, there are eight descriptors that are common to the three approaches, the 39 descriptors, 321 descriptors selected by the CfsSubsetEval routines and the 200 most important descriptors selected by RF algorithm, namely: five topological descriptors (AATS2v, GATS2v, JGI3, topoDiameter, and VR2_Dzp (a Barysz matrix descriptor)) and three count type descriptors (CIC3, BIC1 and RotBFrac (fraction of rotatable bonds, excluding terminal bonds)). The autocorrelation topological descriptors are very relevant for modelling the crystallizability and they are general indices of spatial autocorrelation of distance-type function. The importance of these descriptors (AATS2 $v$ and GATS2v) for the three classes (A, crystalline, $B$, polymorph and $C$, amorphous) is roughly the same, but with a small emphasis on B class for both.

In the work of Wicker \& Cooper $^{1}$ the size descriptor, ${ }^{0} \chi^{\mathrm{v}}$ index (the zero order molecular valence connectivity index), was also reported as been very relevant for modelling the crystallisability of organic molecules. With 1D\&2D set of descriptors, size descriptors such as their topological counterpart appear also to be very relevant for modelling the crystallisability e.g. AATS2v and GATS2v (distance-type function), VR2_Dzp (topological distance matrix), topoDiameter (maximum atom eccentricity). Unlike the autocorrelation descriptors, the VR2_Dzp descriptor seems to be more relevant in A class discrimination and the topoDiameter descriptor in $\mathrm{B}$ class discrimination. In general, the rotatable bonds count is one of descriptors that is established to codify the flexibility of a molecule, and was already reported to be very relevant for modelling the crystallisability of organic molecules, ${ }^{1,2}$ although in our work this descriptor was not selected by the 
CfsSubsetEval routine in the 321 1D\&2D + RDF descriptor set, were selected two related descriptors, RotBFrac (the $40^{\text {th }}$ most important descriptor) and RotBtFrac (fraction of rotatable bonds, including terminal bonds, $67^{\text {th }}$ ). However, the flexibility can also be codified by other descriptors such as descriptors related with chaining (the length $L$ of the longest chain in a molecule; e.g. nAtomLAC, in the 39 descriptors selected by the CfsSubsetEval routine and in the 200 most important descriptors selected by RF algorithm) and branching (e.g. SpMAD_DzZ, SpMAD_Dzm, SpDiam_Dzm, SpDiam_Dzi, in the 321 descriptors selected by the CfsSubsetEval routine and in the 200 most important descriptors selected by RF algorithm).

The four structural categories, e.g. acyclic, heterocyclic, isocyclic and others, were set up in the Reaxys ${ }^{\circledR}$ database for the training and test sets and the Markush structures of the acyclic, heterocyclic and isocyclic categories were represented in Fig. 1. The Markush or generic structures of each structural category were set up using the Markush Editor version 19.11 (ChemAxon Ltd., Budapest, Hungary).

a)

Markush acyclic structures, 512 chemical structures out of 858 molecules

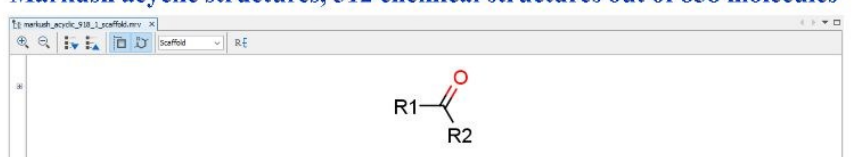

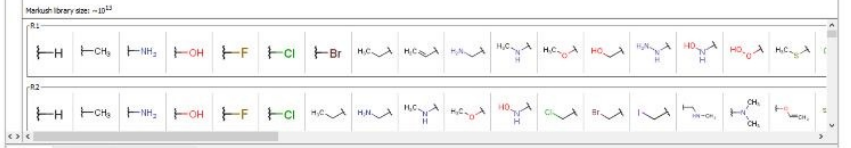

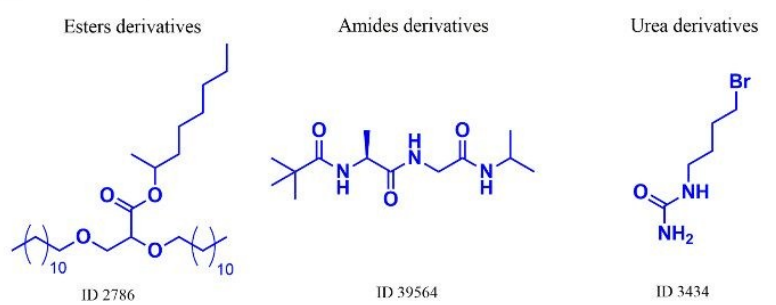

b)

Markush heterocyclic structures, 3433 chemical structures out of 7941 molecules
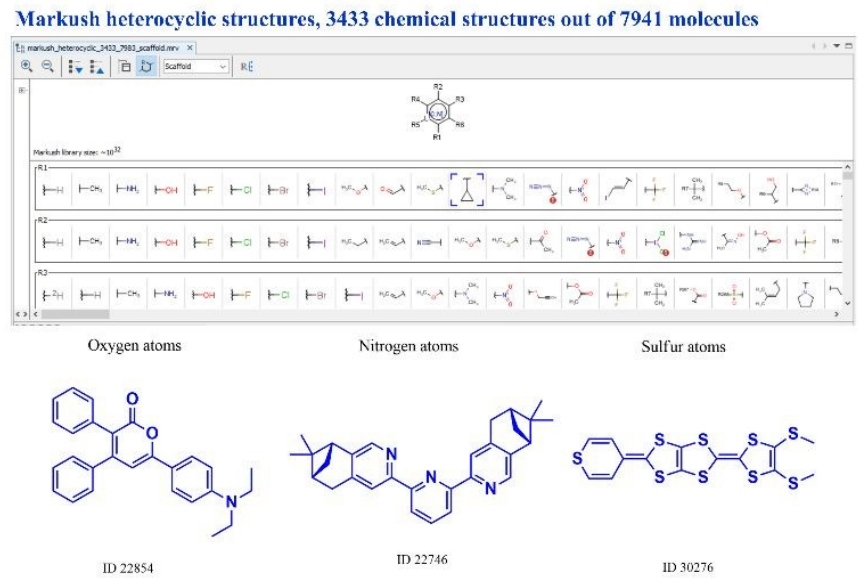

c)

View Article Online Markush isocyclic structures, 2945 chemical structures out 8 Pि1568mbîce | Polycyclic aromatic hydrocarbon

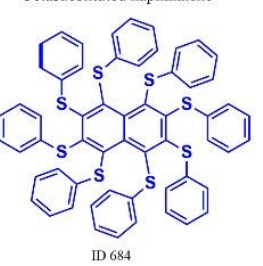

Bicyclic benzene derivative

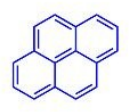

ID 16

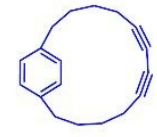

11) 163

Fig. 1 The Markush structures for: a) acyclic, with three examples; b) heterocyclic, with three examples comprising oxygen, nitrogen and sulfur atoms; and c) isocyclic categories of the test set.

In order to establish a few simple rules that provide insights into the crystallisability of organic molecules, a classification tree (CT) model was built with the descriptors 1D_2D + RDF derived with the REPTree algorithm in Weka (see a graphical representation of CT first two rules in Fig. 2 for the test set).

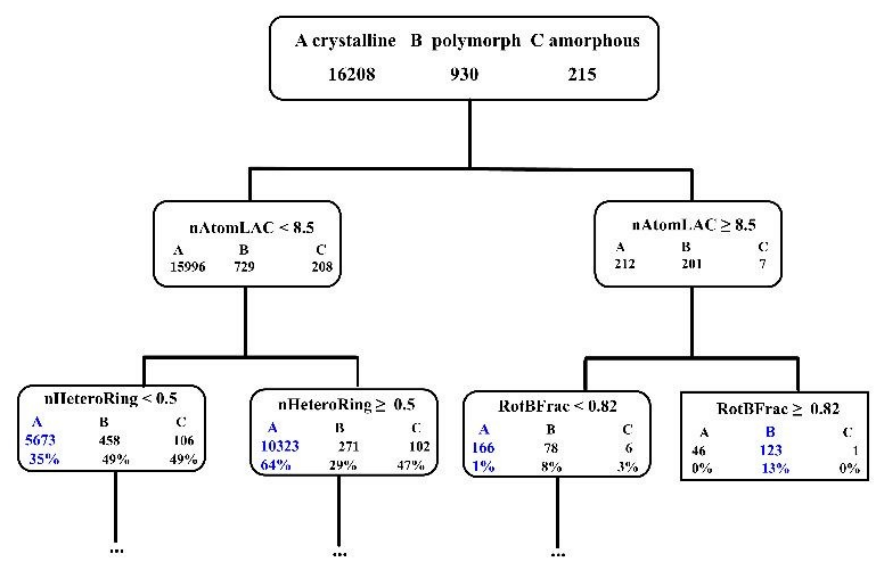

Fig. 2 Representation of the first two rules of the crystallisability classification tree derived with the REPTree algorithm for the test data set.

Using the first and second rules of the CT (nAtomLAC $\geq 8.5$ and RotBFrac $\geq 0.82$ ) it was possible to correctly discriminate 123 polymorphic molecules, corresponding to $13 \%$ of the $B$ class molecules in the test set. From those $B$ class molecules, 115 are acyclic molecules, 5 isocyclic molecules, and 3 heterocyclic molecules. Using the first and other second rules of the CT (nAtomLAC $\geq 8.5$ and RotBFrac < 0.82), 166 molecules are correctly discriminated as belonging to the A Class (crystalline), of which 40, 29, 54 and 43 are acyclic, heterocyclic, isocyclic and other molecules, respectively, as opposed to the molecules of the class B which are mostly acyclic. 
Generative Topographic Mapping (GTM) ${ }^{28}$ software is a probabilistic topology-preserving dimensionality reduction method, which projects the D-dimensional chemical space onto a two-dimensional space that can be exploited for chemical data visualization, ${ }^{29}, 30$ data characterization, comparison or classification ${ }^{29}$ and regression model building. ${ }^{31}$ The training and test sets are represented in 321, 1D\&2D + RDF, descriptor space. A flexible 2D manifold is injected into that space and adapts itself to the training set using 25 nodes (number of grid points, e.g. samples of the probability distribution), 25 RBF (Radial Basis Functions) centres (e.g. traits of the latent probability distribution), 1.5 for $\mathrm{w}$ (width basis for radial basis functions) and 100 for I (regularization coefficient) using the GTMapTool (Varnek Chemoinformatic Laboratory, Strasbourg University, France). The molecules of training set are nonlinearly projected onto the curved manifold which is then
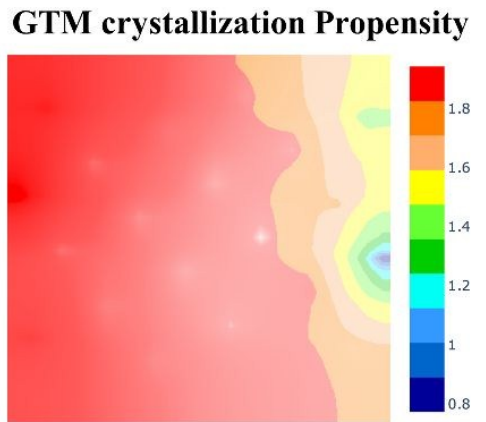

GTM RotBFrac

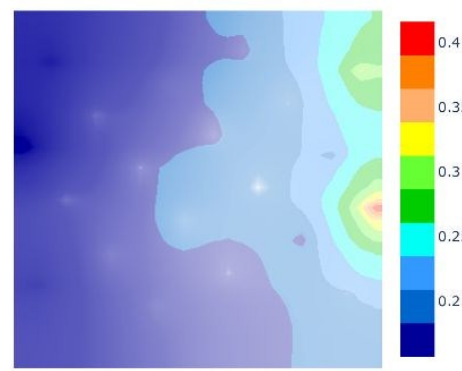

GTM JGI3

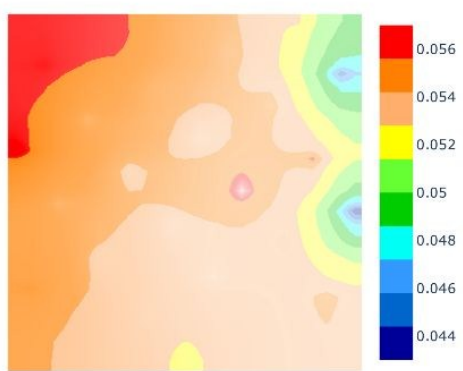

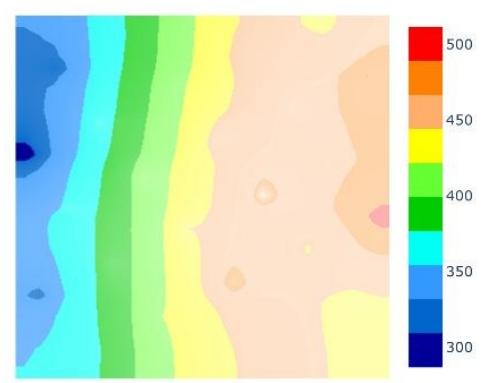

mapped onto a square grid of nodes. Molecules are, however, associated to each node with a certain problabiliejpbased oppthe distance to this node - responsibility using GTMapTool (Varnek Chemoinformatic Laboratory, Strasbourg, France). These responsibilities can be used for both visualization and modelling. In the current work, the projected molecules were visualized on the manifold calculated for the training set, the landscape for the test set was generate in accordance with three classes, crystallines ( 2 class), polymorphics ( 1 class), and amorphs (0 class) using GTMReg (Varnek Chemoinformatic Laboratory, Strasbourg, France), Fig. 3. In order to better characterize the latent space zones underlying these density distributions, some property landscapes such as molecular weight (MW), TopoDiameter, RotBFrac, nAtomLAC, nHeteroRing, JGI3, WTPT-4, and RDF_np_8 were prepared and presented in Fig. 3.

\section{GTM TopoDiameter}

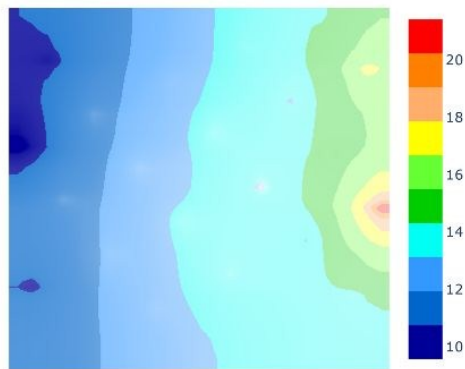

GTM nHeteroRing

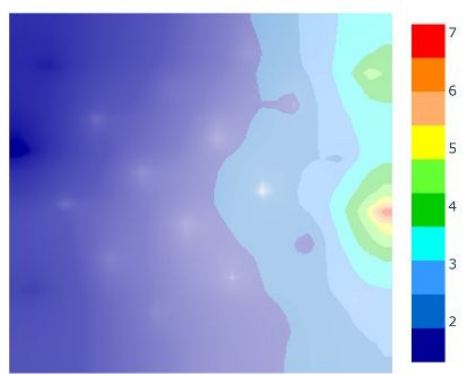

GTM WTPT-4
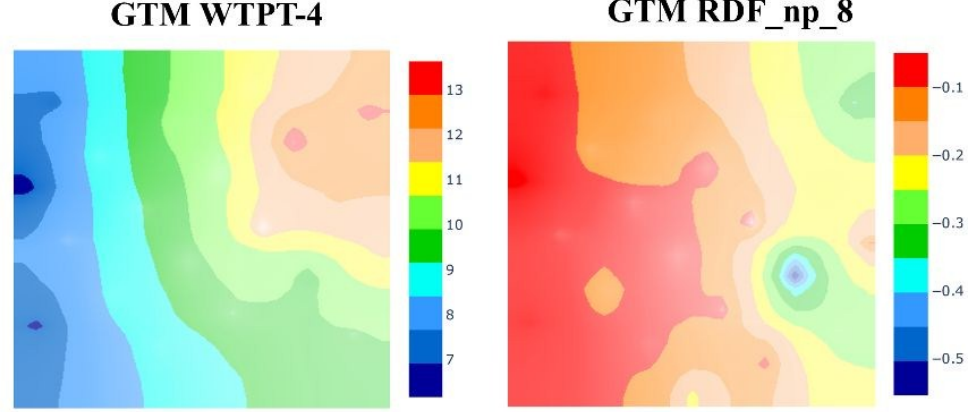

Fig. 3 GTM crystallization propensity landscape for the test set; GTM property landscapes for MW, TopoDiameter, RotBFrac, nAtomLAC, nHeteroRing, JGI3, WTPT-4, and RDF_np_8 of the test set.

MW gradually increases from the left to the right of the map - unsurprisingly, since size is a key parameter of crystallisability chemical space. The TopoDiameter, RotBFrac, the nAtomLAC and WTPT-4 follow this trend. The density of molecules with high MW, TopoDiameter, RotBFrac and nAtomLAC is particularly high on the right side of the map in the region where classes $B$ and $C$ have been mapped (Fig. 3). Interestingly, the descriptors encoding atomic charges JGI3 and RDF_np_8 follow the reverse trend i.e. gradually increase from right to left. In the case of the JGI3 descriptor, the density of the molecules with the lowest values of this descriptor are mapped on the right side 
of the map where classes B and C were mapped. The nHeteroRing descriptor presents a completely different behaviour, the density of the molecules with the highest values of this descriptor are mapped in the central range of the map, and on the left and right ends are mapped to those with the lowest values.

The best model was accomplished with RF algorithm using the 321 selected descriptors, which achieved a G-Mean of 0.488 for the external test set. Majority voting predictions (consensus) obtained by RF, SVM, and MLP models (consensus model, CM) further improved the results with a G-Mean of 0.491 for the test set, Table 6 . The results obtained by the $\mathrm{CM}$ for test set in accordance with the four structural categories (acyclic, heterocyclic, isocyclic and others) that were set up in the Reaxys $^{\circledR}$ database are shown in Table 6 and the Markush structures of the acyclic, heterocyclic (heteroc.) and isocyclic (isoc.) categories were represented in Fig. 1.

Table 6 Performance of the CM by the four structural categories for the test set

\begin{tabular}{|c|c|c|c|c|c|}
\hline CM & All & Acyclic & Heteroc. & Isoc. & Others \\
\hline \# & 17353 & 858 & 7941 & 4568 & 3986 \\
\hline $\mathrm{TA}^{\mathrm{a}}$ & 13159 & 331 & 6779 & 2639 & 3410 \\
\hline $\mathrm{TB}^{\mathrm{b}}$ & 522 & 180 & 72 & 260 & 10 \\
\hline $\mathrm{TC}^{\mathrm{c}}$ & 127 & 6 & 64 & 57 & --- \\
\hline$F A B^{d}$ & 341 & 11 & 179 & 147 & 4 \\
\hline FAC & 60 & 2 & 36 & 22 & --- \\
\hline FBA $^{f}$ & 2023 & 267 & 366 & 1094 & 296 \\
\hline $\mathrm{FBCg}$ & 28 & 2 & 5 & 21 & --- \\
\hline FCA $^{h}$ & 1026 & 55 & 413 & 293 & 265 \\
\hline $\mathrm{FCB}^{\mathrm{i}}$ & 67 & 4 & 27 & 35 & 1 \\
\hline$S E^{j}$ & 0.812 & 0.507 & 0.897 & 0.655 & 0.859 \\
\hline SP_B' & 0.561 & 0.923 & 0.259 & 0.588 & 0.667 \\
\hline SP_Cm & 0.591 & 0.600 & 0.610 & 0.570 & --- \\
\hline$Q^{n}$ & 0.796 & 0.603 & 0.871 & 0.647 & 0.858 \\
\hline G-Meano & 0.491 & 0.514 & 0.472 & 0.470 & 0.537 \\
\hline
\end{tabular}

aTrue A. ${ }^{\mathrm{b}}$ True B. ${ }^{\mathrm{C}}$ True $\mathrm{C}$. ${ }^{\mathrm{d}}$ False $\mathrm{A}$ that was B. ${ }^{\mathrm{e}} \mathrm{False} \mathrm{A}$ that was $\mathrm{C}$. ${ }^{\mathrm{f}} \mathrm{False} \mathrm{B}$ that

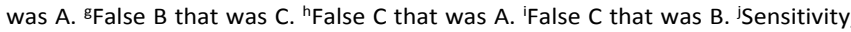
the ratio of true $A$ to the sum of true $A$ and false $A$. 'Specificity $B$, the ratio of true $B$ to the sum of true $B$ and false $B$. mSpecificity $C$, the ratio of true $C$ to the sum of true $C$ and false $C$. " Overall predictive accuracy, the ratio of the sum of true $A$ true $B$ and true $C$ to the sum of true $A$, true $B$, true $C$, false $A$, false $B$ and false $C$. ${ }^{\circ} \mathrm{G}$-mean, the average of G-mean A, G-mean B and G-mean C, where G-mean $x$ is the square root of the product of precision $\mathrm{x}$ and recall $\mathrm{x}$.

Interestingly, the worse predictions obtained considering the G-Mean value for all the structural categories in the test set were to the isocyclic and heterocyclic categories with a G-Mean of 0.470 and 0.472 , respectively, which compares with a GMean of 0.491 for the whole test set. The lower predictive capacity of the CM to the isocyclic and heterocyclic categories appear to be related to the relative low prediction of class $A$ (crystalline) and class B (polymorphic), with a SE of 0.655 and SP_B of 0.259 which compare with the value of 0.812 and 0.561 for the whole test set, respectively. Conversely, the best predictive capacity obtained for the acyclic structural category (G-Mean of 0.514) appears to be related to the high predictive capacity obtained for B class, polymorphic, with SP_B of 0.923 .
The CM was used to predict the crystallisability of another external test set comprising a library0of $10402 \% /$ SQ6stieuted acylanilide derivatives with experimental crystallization propensities, ${ }^{13}$ test set I, Fig. 4.

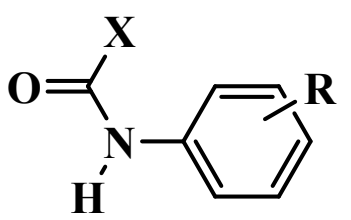

Fig. 4 The test set I includes 402 acylanilide derivatives, in which $\mathrm{X}=\mathrm{H}, \mathrm{CH}_{3}, \mathrm{C}_{2} \mathrm{H}_{5}, \mathrm{C}_{3} \mathrm{H}_{7}, \mathrm{C}\left(\mathrm{CH}_{3}\right)_{3}, \mathrm{CF}_{3}, \mathrm{OCH}_{3}, \mathrm{OC}_{2} \mathrm{H}_{5}, \mathrm{NH}_{2}$ and $\mathrm{Cl}$;

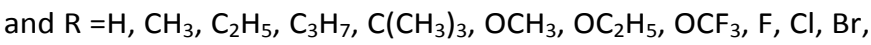
I, $\mathrm{CF}_{3}, \mathrm{OH}, \mathrm{NH}_{2}, \mathrm{COOH}$, etc.

The test set I contains 205 acylanilide derivatives of A class and 197 acylanilide derivatives of B or C classes. For class A were considered only the acylanilide derivatives reported with crystal structure obtained. ${ }^{13}$ For classes B or C were considered all acylanilide derivatives reported as synthesized, but not yet crystallized and with wrong crystal structure obtained, and also the derivatives awaiting crystal structure determination. The crystallisability of test set I was predicted with a $Q$ and G-Mean of 0.542 and 0.540 , respectively, and 127 acylanilide derivatives belonging to the A class were predicted as TA, however only 91 acylanilide derivatives from the $\mathrm{B}$ or $\mathrm{C}$ class were predicted as TB or TC. These results compared with those reported by Bhardwaj et al. a $Q$ of 0.67 for the training set in $O O B$ estimation, ${ }^{11}$ however, there are some differences, our test set I has 402 derivatives according to the original data ${ }^{13}$ while the work reported by Bhardwaj et al. reports 382 acylanilide derivatives. Another important difference is what is established for each class, crystalline and non-crystalline - for the crystalline class (A) only the acylanilide derivatives reported with the obtained crystalline structure are considered, i.e. 205 derivatives, while in the work reported by Bhardwaj et al. there were 303 derivatives (also include derivatives awaiting determination of the crystalline structure). ${ }^{11}$ The various para, meta- or ortho-substituted acylanilide derivatives were similarly predicted by the consensus model, with the hit percentage for A class being of $60 \%, 60 \%$ and $71 \%$, respectively. Acylanilide derivatives substituted at para-, meta- or orthopositions were correctly predicted as belonging to class $B$ with similar percentages, $61 \%, 46 \%$ and $63 \%$, respectively. However, as discussed in the analysis of the descriptors for modelling the crystallization propensity of small organic molecules, the conformation-related parameters appear to be extremely relevant and thus it appears that when the substituent X (Fig. 4) is more rigid and bulky such as $t$-butyl or trifluoromethyl the percentage of hit in the prediction of A class is quite high $(94 \%$ and $100 \%$, respectively). With more flexible substituents where multiple-chain conformations are allowed e.g. ethyl and propyl, the A class hit percentage falls to $52 \%$ and $35 \%$, respectively. 
Another external test set (teste set II) was extracted from the PubChem database, which comprising 198 substances from Crystallography Open Database (http://www.crystallography.net/). This data set was filtered in order to exclude the following atoms: $\mathrm{Ni}, \mathrm{Cd}, \mathrm{Te}, \mathrm{Nd}, \mathrm{Sn}, \mathrm{Co}, \mathrm{Cu}$, $\mathrm{Fe}, \mathrm{Na}, \mathrm{Zn}, \mathrm{Sb}, \mathrm{Pb}, \mathrm{Pn}, \mathrm{Mn}, \mathrm{Tc}, \mathrm{Ga}, \mathrm{V}, \mathrm{Pt}$ and charged molecules, resulting in a test set II with 125 molecules. The crystallisability of test set II was predicted with a Q and G-Mean of 0.704 and 0.839 , respectively, and 88 molecules were predicted as TA. The other molecules ( 37 molecules) were predicted as belonging to the B (32 FB_A) and C (5 FC_A) classes.

Further validation was performed using the $y$ randomization technique. The best RF model using the 321, $1 D \& 2 D+R D F$, descriptors was rebuilt with 3 modified training sets; the $y$-column data (three crystalline classes: A - crystalline; B - Polymorphic; C - Amorphic) was scrambled, keeping the descriptor matrix unchanged. The random models were found to have a considerably lower G-Mean (G-Mean of 0.266-0.339 for the test set) compared to those of the original model (GMean of 0.451-0.491 for the test set, Tables 3-6), further corroborating the statistical reliability of the model.

\section{Conclusions}

The results suggest that the implemented ML models, which were trained with 40,462 molecules (crystallines, A class, 37,790 molecules; polymorphics, B class, 2,167 molecules; and amorphs, C class, 503 molecules), could be used to predict the crystallization propensity of new or existing small organic molecules without crystallisability records, and by this way identify and propose candidate crystalline molecules. The consensus model, obtained by RF, SVM, and MLP models by majority voting predictions, yielded the best model using 321 descriptors included 298 1D\&2D (183 topological and 120 count type descriptors) and 23 RDF 3D descriptors (7 of type a, 7 of type $b$, and 9 of type $c$ ), with $Q$ and G-Mean of 0.80 and 0.49 for an external test set, respectively.

\section{Conflicts of interest}

There are no conflicts to declare.

\section{Funding Sources}

Financial support from Fundação para a Ciência e Tecnologia (FCT) Portugal, under grant UID/QUI/ 50006/2019 (provided to the Associate Laboratory for Green Chemistry LAQV) is greatly appreciated. Florbela Pereira thanks to Fundacão para a Ciência e a Tecnologia, MCTES, for the Norma transitória DL 57/2016 Program Contract.

\section{Acknowledgements}

We thank ChemAxon Ltd. for access to JChem and Marvin, Molecular Networks GmbH for access to CORINA, Alexandre
Varnek Chemoinformatic Laboratory (Strasbourg FieFrance $_{\text {f }}$ for access to GTM.

DOI: 10.1039/DOCE00070A

\section{References}

1. J. G. P. Wicker and R. I. Cooper, Crystengcomm, 2015, 17, 1927-1934.

2. B. C. Hancock, J. Pharm. Sci., 2017, 106, 28-30.

3. J.-M. Nunzi and O. Lebel, Chem. Rec. (New York, N.Y.), 2018.

4. B. Van Eerdenbrugh, J. A. Baird and L. S. Taylor, J. Pharm. Sci., 2010, 99, 3826-3838.

5. K. Nurzynska, J. Booth, C. J. Robert, J. McCabe, I. Dryden and P. M. Fischer, Mol. Pharm., 2015, 12, 3389-3398.

6. K. Nurzynska, R. P. Austin, P. M. Fischer, J. Booth and F. Gommer, J. Pharm. Sci., 2016, 105, 1858-1866.

7. D. Mahlin and C. A. S. Bergstrom, Eur. J. Pharm. Sci., 2013, 49, 323-332.

8. J. A. Baird, B. Van Eerdenbrugh and L. S. Taylor, J. Pharm. Sci., 2010, 99, 3787-3806.

9. L. Yu, S. M. Reutzel-Edens and C. A. Mitchell, Org. Process Res. Dev., 2000, 4, 396-402.

10. B. Rupp and J. W. Wang, Methods, 2004, 34, 390-407.

11. R. M. Bhardwaj, A. Johnston, B. F. Johnston and A. J. Florence, Crystengcomm, 2015, 17, 4272-4275.

12. M. Pillong, C. Marx, P. Piechon, J. G. P. Wicker, R. I. Cooper and T. Wagner, Crystengcomm, 2017, 19, 3737-3745.

13. M. B. Hursthouse, L. S. Huth and T. L. Threlfall, Org. Process Res. Dev., 2009, 13, 1231-1240.

14. C. W. Yap, J. Comput. Chem., 2011, 32, 1466-1474.

15. P. Selzer and P. Ertl, QSAR Comb. Sci., 2005, 24, 270-276.

16. Q. Zhang, F. Zheng, R. Fartaria, D. A. R. S. Latino, X. Qu, T Campos, T. Zhao and J. Aires-de-Sousa, Chemom. Intell. Lab. Sys., 2014, 134, 158-163.

17. R: A Language and Environment for Statistical Computing R Foundation for Statistical Computing; http://www.Rproject.org: Vienna, Austria, 2014.

18. M. A. Hall and L. A. Smith, Twelfth International FLAIRS Conference, Orlando, AAAI Press: Menlo Park, C., USA, Ed. Orlando, 1999; pp 235-239.

19. M. Hall, E. Frank, G. Holmes, B. Pfahringer, P. Reutemann and I. H. Witten, SIGKDD Explor. Newsl., 2009, 11, 10-18.

20. S. Jain, E. Kotsampasakou and G. F. Ecker, J. Comput. Aided Mol. Des., 2018, 32, 583-590.

$21 . \quad$ L. Breiman, Mach. Learn., 2001, 45, 5-32.

22. V. Svetnik, A. Liaw, C. Tong, J. C. Culberson, R. P. Sheridan and B. P. Feuston, J. Chem. Inf. Comput. Sci., 2003, 43, 1947-1958.

23. A. Liaw and M. Wiener, $R$ News, 2002, 2, 18-22.

24. C. Cortes and V. Vapnik, Mach. Learn., 1995, 20, 273-297.

25. C.-C. Chang and C.-J. Lin, ACM Trans. Intell. Syst. Technol., 2011, 2.

26. F. Chollet, Keras, GitHub: 2015.

27. M. Abadi, A. Agarwal, P. Barham, E. Brevdo, Z. Chen, C. Citro, G. S. Corrado, A. Davis, J. Dean, M. Devin, S. Ghemawat, I. Goodfellow, A. Harp, G. Irving, M. Isard, Y. Jia, R. Jozefowicz, L. Kaiser, M. Kudlur, J. Levenberg, D. Mane, R. Monga, S. Moore, D. Murray, C. Olah, M. Schuster, J. Shlens, B. Steiner, I. Sutskever, K. Talwar, P. Tucker, V. Vanhoucke, V. Vasudevan, F. Viegas, O. Vinyals, 
P. Warden, M. Wattenberg, M. Wicke, Y. Yu and X. Zheng, arXiv, 2016, 1603.04467.

28. C. M. Bishop, M. Svensen and C. K. I. Williams, Neural

29. N. Kireeva, Baskin, II, H. A. Gaspar, D. Horvath, G. Marcou and A. Varnek, Mol. Inform., 2012, 31, 301-312.

30. H. A. Gaspar, Baskin, II, G. Marcou, D. Horvath and A. Varnek, J. Chem. Inf. Model., 2015, 55, 84-94.

31. N. Kireeva, S. L. Kuznetsov and A. Y. Tsivadze, Ind. Eng. Chem. Res., 2012, 51, 14337-14343. 